\title{
Probing Stress-Induced Grain Boundary Migration and Hypofriction at High Resolution
}

\author{
M.L. Bowers ${ }^{1}$, C. Ophus ${ }^{1}$, A.M. Minor ${ }^{1,2}$, U. Dahmen ${ }^{1}$ \\ ${ }^{1}$ National Center for Electron Microscopy, Molecular Foundry, LBNL, Berkeley, CA 94720, USA \\ ${ }^{2}$ Department of Materials Science and Engineering, University of California, Berkeley, CA 94720, USA
}

The atomic structure of interfaces in crystalline solids strongly affects many macroscopic properties, including strength, ductility, and thermal stability [1]. Therefore, future material development relies on a fundamental understanding of the atomic-scale structural evolution and migration mechanisms of crystalline interfaces in response to external stimuli. Our recent work identifying a mechanism of grain boundary step coalescence at ambient temperature demonstrates the power of coupling aberrationcorrected electron microscopy with atomistic simulation in understanding interface phenomena [2]. Figure 1d shows a color overlay illustrating atomic trajectories during grain boundary motion, highlighting the level of detail with which structural fluctuations can be traced experimentally. However, this methodology has not yet been applied to the observation of grain boundaries under the influence of an external load, where grain boundary migration mechanisms are expected to be quite different.

In this contribution, we investigate the atomic-scale mechanisms of grain boundary motion in bicrystalline $\mathrm{Au}$ thin films. Thermal evaporation of $\mathrm{Au}$ onto a $<100>\mathrm{Ge}$ substrate results in a $\mathrm{Au}$ film with columnar grain structure consisting of two $\langle 110\rangle$ variants rotated $90^{\circ}$ about the common foil normal, as shown in Figure 1b. This is an ideal system for studying complex interface phenomena in fcc materials due to the simplicity and multitude of available grain boundaries and the absence of chemical effects such as segregation. The (110)/(001) tilt boundary is of particular scientific interest due to its incommensurate interface structure, which has been theoretically predicted to exhibit hypofriction. Also known as superlubricity, this absence of static friction has important consequences for many interfacerelated problems, such as grain boundary engineering and understanding fracture processes. The hypofriction property of this facet was recently tested experimentally by in situ compression of nanopillars [4]. The stress response of pillars containing these facets was consistently lower than single crystal pillars, and the overall shape change was in agreement with simulations. However, the low magnification diffraction contrast imaging coupled with the inherent instability of compression tests prevented any observations of the interfacial structure.

To enhance our understanding of these phenomena, we have performed high resolution in situ tensile experiments to probe stress-induced boundary migration and superglide. Low noise isothermal tension experiments were performed using a Hysitron PI-95 actuator with a Push-to-Pull MEMS device in the TEAM I microscope at the National Center for Electron Microscopy. Figure 1a shows a typical device with $\mathrm{Au}$ film overlaid and moving parts cleared away by FIB milling. Individual boundary segments can be isolated in tension either by exploiting the natural structure of the film (e.g. Figure 1c) or by electron beam sputtering. High frame rate imaging was performed during straining and custom MATLAB routines were employed to track and analyze the evolution of the boundary structure. Strain mapping via scanning diffraction was used to quantify strain localization at unique facets and junctions. This methodology has enabled a direct comparison to previous and on-going simulation efforts, which predict strain localization at intersections with free surfaces. To our knowledge, these experiments are the first of their kind and provide valuable insight into stress-assisted interface processes. [5] 


\section{References}

[1] Y. Mishin, M. Asta, and J. Li, Acta Mater. 58, 1117 (2010).

[2] M.L. Bowers, C. Ophus, A. Gautam, F. Lançon, and U. Dahmen, Phys. Rev. Lett. (in press)

[3] T. Radetic, C. Ophus, D.L. Olmsted, M. Asta, and U. Dahmen, Acta Mater. 60, 7051 (2012).

[4] F. Lançon, J. Ye, D. Caliste, T. Radetic, A.M. Minor, and U. Dahmen, Nano Lett. 10, 695 (2010).

[5] Work at the Molecular Foundry was supported by the Office of Science, Office of Basic Energy

Sciences, of the U.S. Department of Energy under Contract No. DE-AC02-05CH11231
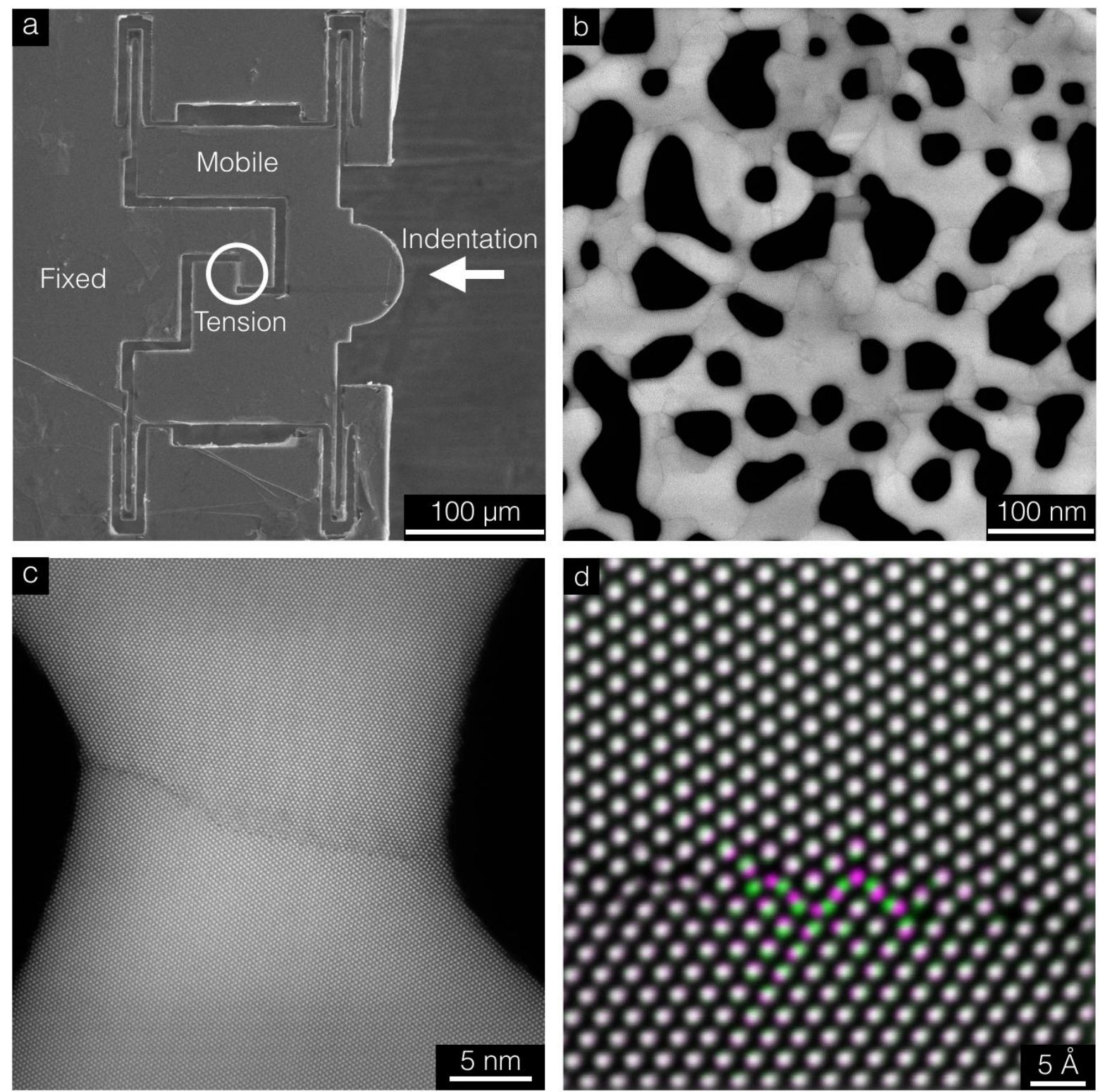

Figure 1: (a) SEM micrograph of a Push-to-Pull MEMS device with Au film overlaid. Film has been removed by FIB milling except the center of the device under tension. (b) HAADF STEM overview of a typical Au bicrystal film. (c) As-deposited tensile "gage section" bisected by an incommensurate grain boundary. (d) Colored overlay showing atomic trajectories during grain boundary motion. 